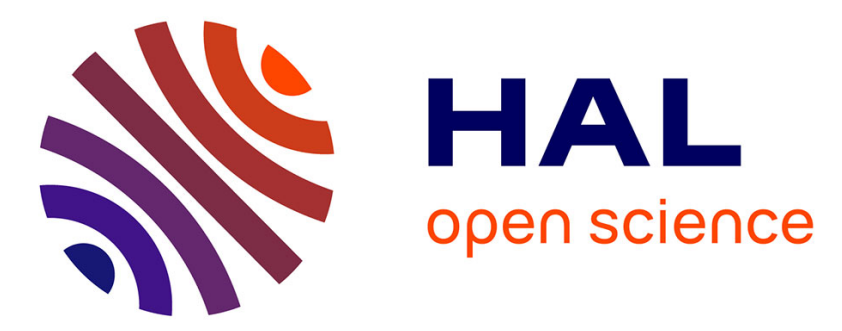

\title{
Texture analysis of lace images using histogram and local binary patterns under rotation variation
}

Wael Ben Soltana, Alice Porebski, Nicolas Vandenbroucke, Adeel Ahmad, Denis Hamad

\section{- To cite this version:}

Wael Ben Soltana, Alice Porebski, Nicolas Vandenbroucke, Adeel Ahmad, Denis Hamad. Texture analysis of lace images using histogram and local binary patterns under rotation variation. 1st International Image Processing, Applications and Systems conference (IEEE-IPAS'14), Nov 2014, Sfax, Tunisia. 10.1109/IPAS.2014.7043325 . hal-03108753

\section{HAL Id: hal-03108753 https://hal.science/hal-03108753}

Submitted on 13 Jan 2021

HAL is a multi-disciplinary open access archive for the deposit and dissemination of scientific research documents, whether they are published or not. The documents may come from teaching and research institutions in France or abroad, or from public or private research centers.
L'archive ouverte pluridisciplinaire HAL, est destinée au dépôt et à la diffusion de documents scientifiques de niveau recherche, publiés ou non, émanant des établissements d'enseignement et de recherche français ou étrangers, des laboratoires publics ou privés. 


\title{
Texture analysis of lace images using histogram and local binary patterns under rotation variation
}

\author{
Wael Ben Soltana, Alice Porebski, Nicolas Vandenbroucke, Adeel Ahmad and Denis Hamad \\ Laboratoire d'Informatique Signal et Image de la Côte d'Opale \\ 50, rue Ferdinand Buisson, BP 719, 62228 Calais Cedex France \\ Email: \{bensoltana, porebski, vandenbroucke, ahmad, denis.hamad\}@lisic.univ-littoral.fr
}

\begin{abstract}
The images of lace textile are particularly difficult to be analyzed in digital form using classical image processing techniques. The major reasons of this difficulty emerge from the complex nature of lace which generally has different textures in its constituents like the background and patterns. In this paper, we study the behavior of Image Histogram (HistI) and Local Binary Patterns $(L B P)$ on image extracts of lace in presence and absence of rotation. We further evaluate two variants of $L B P$; primarily the $L B P$ Histogram $(L B P B)$ and secondly the Fourier Transform applied on the LBP Histogram (LBPFFT). Consequently, we analyze the contribution of data fusion on feature level and score level in the different experimentations. The classification rate evaluates the discrimination degree of each descriptor via the $k$ nearest neighbors $k N N$ classifier. Experimental results indicate that the $L B P B, L B P F F T$ and HistI combined at score level generate the better performance in absence of transformations. Whereas, LBPFFT and HistI combined at the same level generate the better classification rate, in the presence of rotation.
\end{abstract}

Keywords-Lace textile, texture analysis, Local Binary Patterns, Fourier Transform, feature level fusion, score level fusion, kNN classifier.

\section{INTRODUCTION}

Indexing and image retrieval from large databases are being considered as two fields of advanced research. The indexing relates to the extraction of particular signatures, like distribution and features, etc. These describe the content of the image depending on to its texture, color and/or shape. The retrieval (often referred to as search) relates to the comparison of the signature of queried image with the stored signatures to produce the similarity measures. The images which produce the greatest similarity scores are considered as the most similar images in the database. Several applications of indexing and retrieval have been developed on the images of textures [1], faces [2], etc. However in our knowledge, there is no application for indexing and searching images of lace. This may be due to the complex nature of the lace. Indeed, a lace is usually composed of several parts with different textures; for example, background, pattern, etc. In addition, the diversity of lace samples and acquisition conditions may also make it difficult task. Finally, the deformable nature of lace increases this difficulty.

In this paper, we present the results obtained from the studies carried out during the INTERREG IV 2 Seas project CRYSALIS.

978-1-4799-7069-8/14/\$31.00 2014 IEEE
It has the major objective to preserve the justified role of the lace textile in the dedicated history of this field. In this context, we have integrated a search tool, based on lace-contents, in the constructed digital library of lace images. The indexing and retrieval of lace images require an adequate description of theses images by invariant and discriminating descriptors. For this purpose, we separately study the behavior of three types of descriptors of lace images; the Image Histogram(HistI) and two variants of Local Binary Patterns $(L B P)$, which are extracted from the images of lace in the presence or absence of rotation factor. These variants are the Histogram of Basic $L B P(L B P B)$ and its Discrete Fourier Transform (LBPFFT). Later on, we analyze the contribution of data fusion on two levels in the various experiments. The first level (called feature level) operates before the classification of images. It consists of concatenating the descriptors for a single descriptor characterizing the images of lace. The second level (called score level) uses scores generated by different classifiers, which are specific to each descriptor.

The rest of the article is organized as follows: section 2 of this article presents the characteristics of lace subject for image analysis. Section 3 describes the different texture descriptors that we have been testing. Sections 4 and 5 describe the method of classification and fusion information, respectively. Section 6 presents the experimental protocol, while the results are discussed in the section 7. The last section concludes the paper along with future perspectives.

\section{CHARACTERISTICS OF THE IMAGES OF LACE}

The different lace images (also called lace samples) have been taken from the Calais' International Center for Lace and Fashion, France. The original lace designs are scanned as colored images of 600 dpi resolution. The lace samples vary according to several criteria, for example the type of patterns (floral, animal, etc.), the nature of fabric (cotton, textile, etc.), the type and color of background, as explained in the Fig. 1.

This diversity has a common specificity. Indeed, there is a limited and well-defined background lace set (as referred in the Fig. 2). We, initially, attempt to recognize the different types of lace according to their background, which has been a real challenge. These different textures are sometimes very similar and it is difficult to distinguish them among certain background classes. Fig. 2 shows four examples of lace backgrounds where the similitude is observable between ((a), (b)) and ((c), (d)). 
The deformable nature of the lace equally this complexity. In addition, it may have varying acquisition conditions.

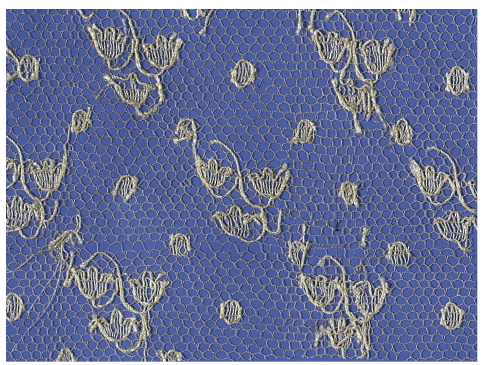

(a) A lace image with white background and some patterns

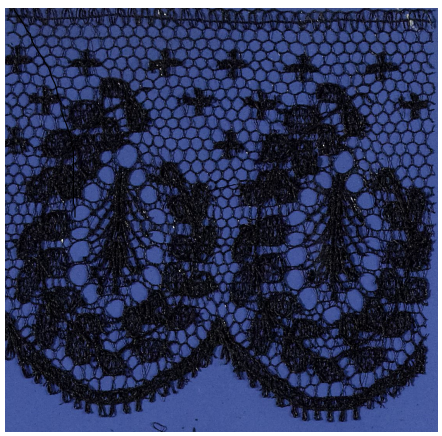

(b) A lace image with black background and some patterns

Fig. 1. Example of lace images

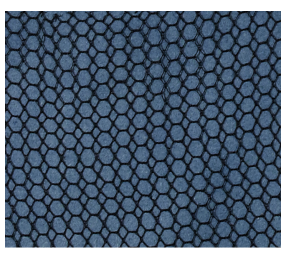

(a)

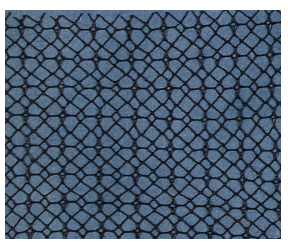

(c)

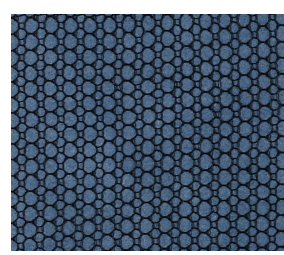

(b)

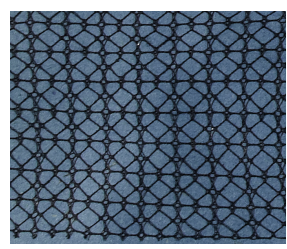

(d)
Fig. 2. A similitude among different lace backgrounds

\section{FEATURE EXTRACTION}

We explored a set of features extracted from lace images, such as gray level lace Image Histogram(HistI), LBP Histogram $(L B P B)$ and the applied Fourier Transform on LBP Histogram (LBPFFT). $k N N$ classifier is applied for all types of features with $k=1$. Indeed, with this classifier, there is no parameter to tune and it is easy to implement.

\section{A. Local Binary Patterns Histogram}

The Local Binary Patterns (LBP) has been proposed by Ojala [3],[4]. The idea of this texture operator is to assign each pixel a specific code depending on the levels of its neighborhood. The level of the centered pixel $\left(i_{c}\right)$ with coordinate $\left(x_{c}, y_{c}\right)$ is compared to its neighbors $\left(i_{n}\right)$ based on the following equation(1):

$$
\begin{gathered}
\operatorname{LBP}\left(x_{c}, y_{c}\right)=\sum_{m=0}^{p-1} s\left(i_{n}-i_{c}\right) \times 2^{n} \\
s\left(i_{n}-i_{c}\right)=1 \quad \text { if } \quad i_{n}-i_{c} \geq 0 \\
0 \quad \text { if } \quad i_{n}-i_{c}<0
\end{gathered}
$$

where, $p$ is the number of neighboring pixels. We consider a $3 x 3$ pixel neighborhood ( $p=8$ neighbors). We obtain a matrix containing the values of $L B P$ between 0 and 255 for each pixel. Next, an histogram is calculated based on these values to construct $\angle B P B$ descriptor .

\section{B. The Fourier Transform on LBP Histogram}

In the literature, [5], [6], the Discrete Fourier transform $(F F T)$ is applied to the $L B P$ histogram. The interest is to have a texture descriptor invariant to rotation transformation. The coefficients of FFT H2(t) can be represented by the following equation(2):

$$
\begin{aligned}
H 2(t) & =\sum_{i=1}^{N} h(i) \times \exp ^{(-j 2 \pi(t-1)(i-1) / N)} \\
1 & \leq t \leq N
\end{aligned}
$$

where, $h$ is the $L B P$ histogram and $N$ is the dimension.

Thereafter, the amplitude of the coefficients of Discrete Fourier transform is selected according to the following equation (3) :

$$
\left|H_{F F T}(t)\right|=\sqrt{H 2(t) * \overline{H 2(t)}}
$$

where, $\overline{H 2(t)}$ denotes the complex conjugate of $H 2(t)$.

These calculated values are used to obtain the $L B P F F T$ descriptor .

\section{ClassificAtion OF LACE IMAGES}

After feature extraction phase, we classify the lace images based on different descriptors. We considered the $k$-nearest neighbor $(k N N)$ due to its effectiveness and flexibility in the experimental protocols [7], [6]. In this context, we build a learning image database. In presence of test image $I$, the $k N N$ method considers the $k$ training images which are nearest to the image by the defined distance. In this paper, we consider the first class with the minimum distance from the image $I(k=1)$. The distance $L 1$ (Eq.4) is chosen [7], [5], [6] to calculate the distance between two vectors $x$ and $y$.

$$
d(x, y)=\sum_{j=1}^{B}\left|\left(x_{j}-y_{j}\right)\right|
$$

where, $x=\left(x_{1}, \ldots, x_{B}\right), y=\left(y_{1}, \ldots, y_{B}\right)$, and $B$ is the dimension. 


\section{FEATURE LEVEL AND SCORE LEVEL FUSION STRATEGIES}

In the literature of pattern recognition and computer vision, there are many fusion-related research works on different levels. Fusion information is a relatively understudied problem because of practical difficulties. It may cause significant classifier performance losses if the best fusion scheme is not appropriately chosen [8]. Several fusion strategies can be roughly classified into two main categories [9], given as follows:

- fusion at an early stage (feature level fusion)

- fusion at a later stage (score level fusion [10],[11]).

In the first category, all vectors of descriptors are concatenated to be classified. For this, a normalization phase is applied to map all features into common scale and range. In this work, L1-norm (equation 5) has been considered.

$$
x^{\prime}=\frac{x}{\|x\|_{1}}
$$

where, $x$ is a feature vector and $x^{\prime}$ is the normalized vector.

In score level fusion, each classifier operates independently, then all decisions or distances are fused. In fact, each classifier receives as input a feature vector $x$ and generates a set of outputs $e_{j}(x)$ (equation 6).

$$
e_{j}(x)=\left[d_{j}^{1}, d_{j}^{i}, . ., d_{j}^{C}\right]
$$

where, $d_{j}^{i}$ is generated by the classifier $j$. It is attributed to the class $i$, and $C$ is the total number of classes in the training set.

However, all scores must be normalized in the same range [8] [12]. In this work, max-min (equation 7) has been applied on $e_{j}$ to produce a new vector $e_{2 j}=\left[d_{2 j}^{1}, d_{2 j}^{i}, \ldots, d_{2 j}^{C}\right] . d_{2 j}^{i}$ can be defined as follows:

$$
d_{2 j}^{i}=\frac{d_{j}^{i}-e_{j, \text { min }}}{e_{j, \max }-e_{j, \min }}
$$

where, $e_{j, \max }$ and $e_{j, \min }$ are maximum and minimum values of the vector $e_{j}$, respectively.

Next, a sum rule [13] [11] is adopted to calculate the global distance $S_{i}$ for each class in learning database using equation 8 .

$$
S_{i}=\sum_{j=1}^{K} d_{2 i}^{j}
$$

where, $K$ is the number of classifiers. For each test image $I$, a decision rule consider the best class $C_{i}$ with the smallest distance $S_{i}$, and $i$ can be defined as follows:

$$
i=\operatorname{argmin}_{i=1, \ldots C}\left(S_{i}\right)
$$

\section{EXPERIMENT PROTOCOL}

In this section, we briefly discuss the used experimental protocols for the decomposition of the image database to conduct all tests. Initially, the image database consisted of 492 images obtained from 41 lace backgrounds. Each lace background image generates 12 thumbnails with fixed size $(150 * 150$ pixels $)$.
The database is essentially divided into two subsets. The subset 1 is composed of the learning database and test database. In subset 2 , we add new test images which correspond to the original test images where rotations of $90^{\circ}, 180^{\circ}$ and $270^{\circ}$ are applied.

All images of the database are presented in the same scale. Table I presents the whole subsets. Subset 1 has been tested without transformation, whereas the Subset 2 assesses the impact of rotation transformation on the performance of all descriptors.

TABLE I. EXPERIMENT SETTINGS

\begin{tabular}{|l|c|c|}
\hline Number of subset & $\begin{array}{c}\text { Size of learning } \\
\text { subset }\end{array}$ & $\begin{array}{c}\text { Size of test } \\
\text { subset }\end{array}$ \\
\hline \hline 1 & $246\left(6^{*} 41\right)$ & $246\left(6^{*} 41\right)$ \\
\hline 2 & $246\left(6^{*} 41\right)$ & $984\left(4 * 6^{*} 41\right)$ \\
\hline
\end{tabular}

The classification rate is adopted as performance metric. It represents the number of test images correctly classified on the total number of test images. For each subset, we applied a cross-validation with around 20 experiments. In each experiment, we randomly selected 6 training images and 6 test images for each image of lace background. We adopt the average classification rate in all experiments. The descriptor level fusion and score level fusion are noted as Fusion I and Fusion II, respectively.

\section{EXPERIMENTAL RESULTS}

The experimental results provides a means to observe the behavior of the descriptors. We discuss, in below, the obtained results without and with the rotation transformation.

\section{A. Results without transformation}

All images in subset 1 are used to assess all descriptors as well as all different fusion schemes. We evaluate the combination of fusion schemes of the three descriptors (HistI, $L B P B, L B P F F T)$. Table II presents all classification rates.

TABLE II. CLASSIFICATION RATES WITH SUBSET 1 IN FUSION I AND FUSION II

\begin{tabular}{|l|c|c|}
\hline & Fusion I & Fusion II \\
\hline \hline HistI & 0.7348 & 0.7348 \\
\hline LBPB & 0.9705 & $\mathbf{0 . 9 7 0 5}$ \\
\hline LBPFFT & 0.8689 & 0.8756 \\
\hline \hline LBPB + HistI & 0.9163 & 0.9657 \\
\hline LBPB + LBPFFT & 0.9669 & 0.9648 \\
\hline HistI + LBPFFT & 0.8774 & 0.9246 \\
\hline LBPB + HistI + LBPFFT & 0.9443 & $\mathbf{0 . 9 7 3 2}$ \\
\hline
\end{tabular}

The first three lines show the individual classification rate of the three descriptors. We detect a slight decrease in performance for the LBPFFT descriptor in the Fusion I because of the applied normalization operation. We observe that $L B P B$ generates better results with the classification rate $97.05 \%$, followed by the performance of $L B P F F T$ with $86.98 \%$ in Fusion I and $87.56 \%$ in Fusion II. It can be helpful to note that the local binary patterns yield an increase of $24 \%$ compared to the image histogram descriptor. This shows that 
the information of the texture is more discriminating than the information which only analyzes the levels of the pixels without their spatial interaction.

In the feature fusion level, we did not find a fusion scheme for the improvable performance to be achieved by the $L B P B$. It is notifiable due to the poor performance obtained by HistI and $L B P F F T$ as compared with the performance of the $L B P B$. On the other side, the concatenation of two descriptors HistI and $L B P F F T$ improves the classification rate of $13 \%$ with respect to the individual performance of the descriptor HistI and $1 \%$ compared with the individual performance of $L B P F F T$ descriptor. This improvement highlights the complementarity between these two descriptors.

In the fusion score level, all performances (Fusion II) are presented in table II. We note that the only fusion scheme that combines three descriptors leads to a slight improvement of the performance of $\angle B P B$ descriptor. In this case, we also note an improvement of about $0.27 \%$ of the classification rate. This small increase may be partially due to the simple sum rule (Eq.8) which is not weighted. Consequently, it provides the same confidence and importance to all descriptors which have distinct performance.

\section{B. Results with rotation transformation}

All images in subset 2 are used to assess all descriptors as well as all different fusion schemes under rotation transformation. The table III provides different results. The histogram image and $\angle B P F F T$ are invariant descriptors to rotation transformation, the obtained classification results are similar to those obtained with the images of the subset 1 . On the other side, we see a negative impact of rotation in the performance of the $\angle B P B$ descriptor. A performance decrease of $49 \%$ is observed in comparison to the experiment 1 . To reduce this negative effect, we perform two fusion levels. In the fusion I, the concatenation of all descriptors achieves $85.01 \%$ of classification rate. It can be observed that the integration of $L B P B$ in the feature fusion strategy does not improve the overall performance as compared to the best evaluated classification rate $(86.89 \%)$ generated by $L B P F F T$. When $\angle B P B$ is not selected, the scheme (HistI $+\angle B P F F T)$ achieves the better overall performance with $87.74 \%$ of classification rate.

TABLE III. CLASSIFICATION RATES IN FUSION I AND FUSION II WITH SUBSET 2

\begin{tabular}{|l|c|c|}
\hline & Fusion I & Fusion II \\
\hline \hline HistI & 0.7348 & 0.7348 \\
\hline LBPB & 0.4731 & 0.4731 \\
\hline LBPFFT & 0.8689 & 0.8756 \\
\hline \hline LBPB + HistI & 0.7172 & 0.5943 \\
\hline LBPB + LBPFFT & 0.5745 & 0.5758 \\
\hline HistI + LBPFFT & 0.8774 & $\mathbf{0 . 9 2 4 6}$ \\
\hline LBPB + HistI + LBPFFT & 0.8501 & 0.7991 \\
\hline
\end{tabular}

In the fusion score level, the same behavior is observed for the $\angle B P B$ descriptor. Indeed, the fusion scheme $L B P F F T$ and HistI gives the best evaluated performance results $(92.46 \%)$. This result agrees on the benefit of fusion at score compared to the fusion descriptor level seen in the two tested scenarios. It is difficult to compare our results with those available in the state of the art since the considered databases are different. However, the obtained results confirm those of Ahonen [5] and Guoying [6] concerning the comparison of rotation invariant attributes in respect of basic attributes.

\section{CONCLUSION}

In this article, we analyze the performance of three descriptors which are the image histogram and two variants of local binary patterns ( $\angle B P B$ and $L B P F F T$ ) extracted from lace images in the presence or absence of rotation transformation. Subsequently, we analyze the contribution of the feature level fusion and score level fusion in the various experiments. The results show that in the absence of transformation, $L B P B$, LBPFFT and HistI merged in score level generate the best classification rate $(97.32 \%)$. In the same fusion level and in presence of rotation transformation, LBPFFT and HistI generate the better classification rate with $92.46 \%$.

In future, we plan to apply multi-resolution $L B P$ to achieve better accuracy using other types of $L B P$ such as $L B P$ uniform and $L B P$ uniform invariant to rotation. Moreover, we will study other fusion methods. We also expect to integrate scale transformation in our experiments.

\section{ACKNOWLEDGMENTS}

This study is performed within the INTERREG IV 2 seas Project CRYSALIS. We acknowledge the participation of project partners (Calais' International Center for Lace and Fashion, City of Calais, France, University of Creative Arts, Plymouth College of Art, England, and Textile Open Innovation Center, RONSE, Belgium).

\section{REFERENCES}

[1] T. Ojala, T. Mäenpää, M. Pietikäinen, J. Viertola, J. Kyllönen, and S. Huovinen, "Outex new framework for empirical evaluation of texture analysis algorithms," In Proceedings of the 16th International Conference on Pattern Recognition, vol. 1, pp. 701-706, 2002.

[2] K. I. Chang, K. W. Bowyer, and P. J. Flynn, "An evaluation of multimodal 2d+3d face biometrics," IEEE Trans. Pattern Anal. Mach. Intell, vol. 27, no. 4, pp. 619-624, 2005.

[3] T. Ojala, M. Pietikäinen, and D. Harwood, "A comparative study of texture measures with classification based on feature distributions," Pattern Recognition, vol. 29, no. 1, pp. 51-59, 2002.

[4] T. Ojala, M. Pietikäinen, and T. Mäenpää, "Multiresolution gray-scale and rotation invariant texture classification with local binary patterns," Pattern Recognition, vol. 24, no. 7, pp. 971-987, 2002.

[5] T. Ahonen, J. Matas, and M. Pietikäinen, "Rotation invariant image description with local binary pattern histogram fourier features," Image Analysis, pp. 61-70, 2009.

[6] Z. Guoying, T. Ahonen, J. Matas, and M. Pietikäinen, "Rotationinvariant image and video description with local binary pattern features," IEEE Transactions on Image Processing, vol. 21, no. 4, pp. 1465-1477, 2012.

[7] T. Ahonen, A. Hadid, and M. Pietikäinen, "Face recognition with local binary pattern," Computer Vision, ECCV, pp. 469-481, 2004.

[8] W. B. Soltana, D. Huang, M. Ardabilian, L. Chen, and C. B. Amar, "Comparison of $2 \mathrm{~d} / 3 \mathrm{~d}$ features and their adaptive score level fusion for 3d face recognition," 3D Data Processing, Visualization and Transmission (3DPVT), Paris, pp. 17-20, May 2010. 
[9] A. Jain, K. Nandakumar, and A. Ross, "Score normalization in multimodal biometric systems," Pattern Recognition, vol. 38, no. 12, pp. 2270-2285, 2005.

[10] A. K. Jain, R. P. W. Duin, and J. Mao, "Statistical pattern recognition: A review," IEEE Transactions on Pattern Analysis and Machine Intelligence, vol. 22, no. 1, pp. 4-37, 2000.

[11] W. B. Soltana, D. Huang, M. Ardabilian, L. Chen, and C. B. Amar, "A mixture of gated experts optimized using simulated annealing for 3d face recognition," International Conference on Image Processing (ICIP), Brussels, pp. 11-14, Septembre 2011.

[12] Y. S. Huang, K. Liu, and C. Y. Suen, "The combination of multiple classifiers by neural network approach," Journal of Pattern Recognition and Artificial Intelligence, vol. 9, no. 3, pp. 579-597, 1994.

[13] J. Kittler, M. Hatef, R. P. W. Duin, and J. Matas, "On combining classifiers," IEEE Transactions on Pattern Analysis and Machine Intelligence, vol. 20, no. 3, pp. 226-239, 1998. 